\title{
Effective density of Aquadag and fullerene soot black carbon reference materials used for SP2 calibration
}

\author{
M. Gysel ${ }^{1}$, M. Laborde ${ }^{1}$, J. S. Olfert ${ }^{2}$, R. Subramanian ${ }^{3}$, and A. J. Gröhn ${ }^{4}$ \\ ${ }^{1}$ Laboratory of Atmospheric Chemistry, Paul Scherrer Institut, 5232 Villigen PSI, Switzerland \\ ${ }^{2}$ Department of Mechanical Engineering, University of Alberta, Edmonton AB T6G 2G8, Canada \\ ${ }^{3}$ Droplet Measurement Technologies, Boulder, CO 80301, USA \\ ${ }^{4}$ Particle Technology Laboratory, Department of Mechanical and Process Engineering, ETH Zurich, Sonneggstrasse 3, \\ 8092 Zurich, Switzerland
}

Received: 25 July 2011 - Published in Atmos. Meas. Tech. Discuss.: 3 August 2011

Revised: 7 December 2011 - Accepted: 19 December 2011 - Published: 22 December 2011

\begin{abstract}
The mass and effective density of black carbon (BC) particles generated from aqueous suspensions of Aquadag and fullerene soot was measured and parametrized as a function of their mobility diameter. The measurements were made by two independent research groups by operating a differential mobility analyser (DMA) in series with an aerosol particle mass analyser (APM) or a Couette centrifugal particle mass analyser (CPMA). Consistent and reproducible results were found in this study for different production lots of Aquadag, indicating that the effective density of these particles is a stable quantity and largely unaffected by differences in aerosol generation procedures and suspension treatments. The effective density of fullerene soot particles from one production lot was also found to be stable and independent of suspension treatments. Some differences to previous literature data were observed for both Aquadag and fullerene soot at larger particle diameters. Knowledge of the exact relationship between mobility diameter and particle mass is of great importance, as DMAs are commonly used to size-select particles from BC reference materials for calibration of single particle soot photometers (SP2), which quantitatively detect the $\mathrm{BC}$ mass in single particles.
\end{abstract}

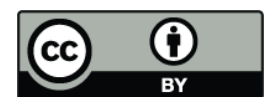

Correspondence to: $\mathrm{M}$. Gysel (martin.gysel@psi.ch)

\section{Motivation}

Black carbon, $\mathrm{BC}$, is the main absorbing component of atmospheric aerosols and estimates show that it may be the second strongest contributor to global warming (Ramanathan and Carmichael, 2008). Traffic-related BC particles have also been shown to cause adverse health effects (e.g. Laden et al., 2000; Slama et al., 2007). Quantitative measurements of BC mass concentrations in atmospheric aerosols are most commonly done with optical, thermal, combined thermaloptical or photo-acoustic methods (Gelencsér, 2004). Recently the single particle soot photometer (SP2; Stephens et al., 2003) was introduced as an alternative method, using laser-induced incandescence (LII) for quantitative detection of refractory BC mass. All BC measurement methods have their advantages and disadvantages, though recent studies showed that consistent results can be obtained for BC measured by different methods, if appropriate calibrations, sampling techniques and data analysis procedures are applied (e.g. Kondo et al., 2011).

The SP2 is a highly sensitive method for selective and quantitative detection of refractory $\mathrm{BC}$ in single particles. The peak height of the incandescence signal is nearly linearly dependent on the mass of refractory BC in the particle, largely independent of particle morphology, and without interference from non-refractory coatings (Schwarz et al., 2006; Slowik et al., 2007; Moteki and Kondo, 2007; Cross et al., 2010). Nevertheless, empirical calibration of the SP2 is still required to obtain absolute $\mathrm{BC}$ mass measurements. This is commonly done with $\mathrm{BC}$ reference materials such as

Published by Copernicus Publications on behalf of the European Geosciences Union. 
Aquadag, glassy carbon or fullerene soot. It has been shown that significant differences exist in the SP2's sensitivity to different $\mathrm{BC}$ materials and that fullerene soot behaves most similarly to ambient $\mathrm{BC}$ in terms of SP2 response per unit mass of BC, refractive index and effective density (Moteki and Kondo, 2010; Moteki et al., 2010; Kondo et al., 2011; Laborde et al., 2011b). The calibration curve of the SP2 is obtained by recording the incandescence signal peak height for pure $\mathrm{BC}$ particles of known mass over the whole dynamic range of the SP2. Selecting particles by mass is ideally done with an aerosol particle mass analyser (APM) or a Couette centrifugal particle mass analyser (CPMA). However, most research groups operating an SP2 do not have access to such a device. Thus the calibration particles are often selected by their mobility diameter using a differential mobility analyser (DMA) instead. Unfortunately particles of many BC reference materials are not compact spheres (Moteki et al., 2009), instead possessing a fractal-like or other irregular shape. Thus, the effective density $\left(\rho_{\text {eff }}\right)$, defined as

$$
\rho_{\mathrm{eff}}=\frac{6 m}{\pi D_{\mathrm{mob}}^{3}},
$$

needs to be known in order to calculate the mass $(m)$ of the size-selected particles from their mobility diameter $\left(D_{\mathrm{mob}}\right)$. This particular definition of effective density corresponds to the first variant of effective density discussed in DeCarlo et al. (2004).

Factory calibration of the SP2 is done with Aquadag. The manufacturer also distributes batches of Aquadag, originating from different production lots, to all SP2 users. The sizeresolved effective density of Aquadag and fullerene soot has been measured by Moteki and Kondo (2010). However, little is known about the influence of e.g. batch-to-batch variability on the effective density of these calibration materials. For this reason we present size-resolved effective density measurements of Aquadag and fullerene soot, including different production lots, varied sample suspension treatments, and measurements by two independent research groups.

\section{Experimental methods}

Two independent research groups from the Laboratory of Atmospheric Chemistry at the Paul Scherrer Institut (PSI) and from the Department of Mechanical Engineering at the University of Alberta (UofA) contributed to the effective density measurements presented in this study. Experimental procedures applied by these two groups were similar, though not perfectly equal, as detailed in the following.

\subsection{Aerosol generation}

Several jars of Aquadag ${ }^{\circledR}$ (Aqueous Deflocculated Acheson Graphite) from Acheson Inc., USA, were purchased by Droplet Measurement Technologies. They took small batches from the production lots \#9054 ("classic") and \#9627 ("new bottle") and distributed each to PSI and UofA. Aquadag is a colloidal dispersion of aggregates of irregular flakes of graphite (Moteki et al., 2009) in water $\left(\sim 80 \% \mathrm{H}_{2} \mathrm{O}\right.$ as delivered).

Fullerene soot was obtained from Alfa Aesar (stock \#40971, lot \#FS12S011). This very fullerene soot sample is of particular interest as it was used for several DMA based SP2 calibrations, e.g. during an SP2 intercomparison workshop (Laborde et al., 2011a). Fullerene soot particles are fractal-like aggregates of spherical primary particles with a diameter of $\sim 50 \mathrm{~nm}$ (Moteki et al., 2009) and they are composed of $\geq 90 \%$ amorphous carbon (carbon black) and $\leq 10 \%$ fullerenes (mainly $\mathrm{C}_{60}$ ) according to Alfa Aesar's specifications.

Materials used for SP2 calibration should ideally have an elemental carbon (EC) mass fraction close to $100 \%$ and give similar incandescence signal amplitude per carbon mass in the SP2 as ambient BC. Thermal-optical analysis of bulk samples of dried Aquadag revealed that $\sim 90-93 \%$ of the total carbon (TC) content are EC and $\sim 7-10 \%$ organic carbon (OC). The TC content accounts for $\sim 83 \%$ of the total gravimetrically determined mass, and consequently EC makes up $\sim 76 \%$ of the total mass. Tests with thermodenuding Aquadag particles at $400^{\circ} \mathrm{C}$ before selecting them by mass resulted in $\sim 15 \%$ higher SP2 signal amplitude per particle mass compared to non-thermodenuded Aquadag. This indicates that thermodenuding removes most of the non-EC components, thereby increasing the EC mass fraction. Similarly, thermodenuding also increased the SP2 signal amplitude per particle mass for fullerene soot particles by $\sim 15 \%$. However, here we report effective density measurements of non-thermodenuded Aquadag and fullerene soot for the following reasons: this is the way it has been done in previous studies relating the properties of $\mathrm{BC}$ calibration materials to those of ambient $\mathrm{BC}$ denuded at $400^{\circ} \mathrm{C}$ in Tokyo (Moteki and Kondo, 2010; Moteki et al., 2010) and it is common calibration practice among the SP2 users.

\subsection{Effective density measurements}

The experimental setup (Fig. 1) applied here was used in previous studies for effective density measurements (e.g. Olfert et al., 2007; Scheckman et al., 2009). Aquadag and fullerene soot were suspended in ultrapure water and $\mathrm{BC}$ aerosol particles were generated from the suspension by means of an atomizer connected to a silica gel diffusion dryer and a ${ }^{85} \mathrm{Kr}$-neutralizer. A DMA (TSI model 3080L; Knutson and Whitby, 1975) was then used to size-select BC particles by their mobility diameter, before passing them through an APM (Kanomax Model APM-3600; Ehara et al., 1996) or a CPMA (Olfert and Collings, 2005) for subsequent selection by particle mass. Particle mass analysers select particles by their mass-to-charge ratio by balancing the electrostatic and centrifugal forces which act on particles introduced into a 


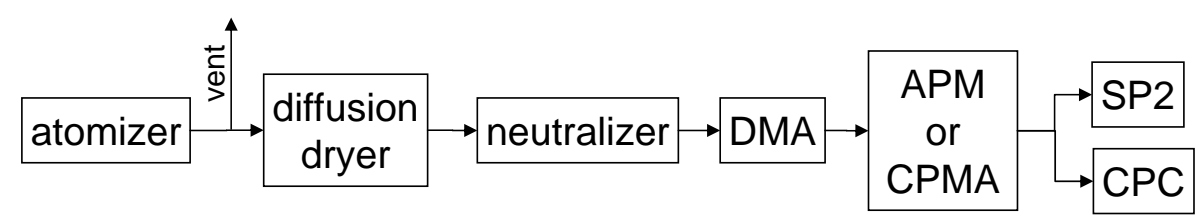

Fig. 1. Experimental setup for effective density measurements.

thin annular space formed between rotating cylindrical electrodes. The mass of particles with a well-defined mobility diameter (fixed DMA voltage) was obtained by stepping the APM (or CPMA) voltage, with simultaneous counting of the particle concentration at the APM (or CPMA) outlet using a condensation particle counter (CPC). Experimental differences between the two research groups contributing to this study were only minor. PSI used a custom-built Collison type atomizer, an APM for mass selection, a TSI CPC model 3022, and additionally an SP2 sampling in parallel to the CPC. UofA used a TSI Collison type atomizer model 3076, a CPMA for mass selection, and a TSI CPC model 3776.

Accurate operation of the DMA+APM and the DMA + CPMA configurations was verified by measuring certified polystyrene microsphere size standards (PSL spheres; Duke Scientific, USA). The effective density determined for the PSL spheres was always found to be within $10 \%$ of the value of $1050 \mathrm{~kg} \mathrm{~m}^{-3}$ stated by the manufacturer. Thus, the accuracy of the effective density measurements is estimated to be $10 \%$ given the diameter uncertainty of the PSL spheres of $\sim 3 \%$.

An example measurement with the DMA-APM system of the effective density of fullerene soot particles with a mobility diameter of $D_{\text {mob }}=456 \mathrm{~nm}$ is shown in Fig. 2. The rotational speed of the APM is held constant and the voltage is stepped. The mass of the monodisperse particles is obtained by fitting the data to find the voltage where the number concentration is at a maximum, $V_{\mathrm{c}}$, and using the following relationship (Ehara et al., 1996),

$m=\frac{q V_{\mathrm{c}}}{\omega^{2} r_{\mathrm{c}}^{2} \ln \left(r_{\mathrm{o}} / r_{\mathrm{i}}\right)}$,

where $q$ is the charge on the particle, $r_{\mathrm{i}}$ and $r_{\mathrm{o}}$ are the inner and outer radii of the cylinders, $\omega$ is the rotational speed of the cylinders, and $r_{\mathrm{c}}=\left(r_{\mathrm{i}}+r_{\mathrm{o}}\right) / 2$. In general, PSI operated the DMA and the APM with resolutions of $0.10-0.23$ and $0.06-0.25^{1}$, respectively, expressed as full width at half maximum (FWHM) in relative terms of the electrical mobility and centre mass. UofA operated the DMA and the

\footnotetext{
${ }^{1}$ At one point the APM was operated at a resolution of 0.46 , which might lead to increased uncertainty in the mass measurement if multiply-charged particles were significant. However, SP2 data was used to verify that multiply-charged particles had an insignificant effect on the APM data fit.
}

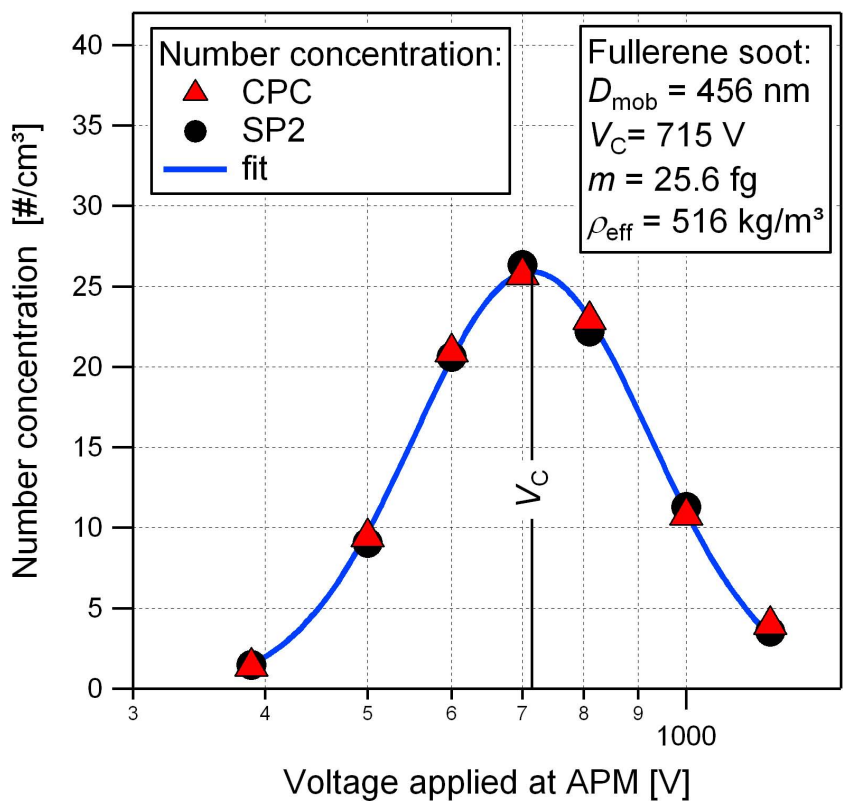

Fig. 2. Classification of fullerene soot particles with mobility diameter $D_{\text {mob }}=456 \mathrm{~nm}$ with the DMA-APM system. Raw number concentration is shown as a function of voltage applied at the APM. The mass, $m=25.6 \mathrm{fg}$, of the monodisperse particles, is obtained from the voltage, $V_{\mathrm{c}}$, where the fitted number concentration is at a maximum (APM operated at $2000 \mathrm{rpm}$ ).

CPMA with FWHMs of 0.10 and 0.15 , respectively. This resolution is sufficiently high such that the mass obtained by fitting the raw distribution agreed with the mass obtained by the application of a more sophisticated charge-correction scheme (Olfert et al., 2007), as multiply-charged particles did not significantly affect the data fit. The SP2 measurements made by PSI downstream of the APM confirmed that all detected particles were $\mathrm{BC}$ particles and that the interference of multiply charged particles was always negligible. The mass measured in the example shown in Fig. 2 was $m=25.6 \mathrm{fg}$ at $D_{\text {mob }}=456 \mathrm{~nm}$, which corresponds to an effective density of $\rho_{\text {eff }}=516 \mathrm{~kg} \mathrm{~m}^{-3}$.

Besides the direct effective density measurement described above, an alternative indirect method was additionally applied to retrieve the effective density of fullerene soot by PSI. For this purpose the SP2 was first calibrated for the 


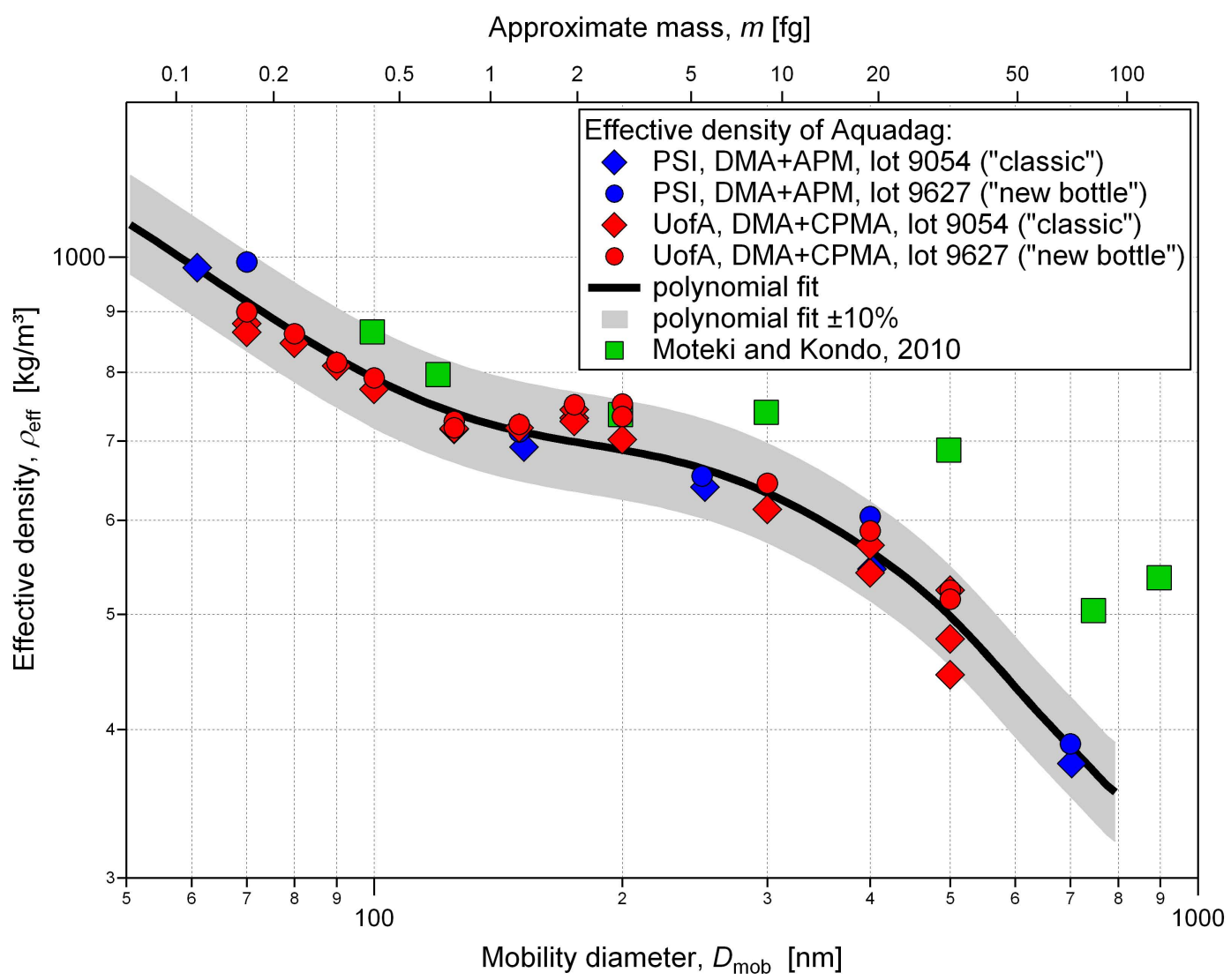

Fig. 3. Effective density of Aquadag as a function of mobility diameter. The approximate mass scale given at the top axis has been calculated from the mobility diameters of the bottom axis using the polynomial fit.

fullerene soot sample using the APM, i.e. a setup as shown in Fig. 1 except without the DMA. After that, size-selected fullerene soot was measured by the SP2, using a setup as shown in Fig. 1 except without the APM. For each selected mobility diameter, the mass measured by the SP2 was determined according to the calibration curve obtained from the previous APM measurement, and the corresponding effective density was then calculated using Eq. (1). Results of this novel approach to measure the effective density of pure BC particles will be shown in Sect. 3.2.

\section{Results and discussion}

\subsection{Aquadag}

The material density of pure graphite is $>2000 \mathrm{~kg} \mathrm{~m}^{-3}$ and the producer of Aquadag states a "density" of $\sim 1120 \mathrm{~kg} \mathrm{~m}^{-3}$ in the product data sheet. Neither value has little to do with the effective density required to calculate particle mass from mobility diameter, as shown in Fig. 3 by the measurements of PSI (blue markers) and UofA (red markers). The effective density of Aquadag decreases from $\sim 1000 \mathrm{~kg} \mathrm{~m}^{-3}$ at
$D_{\mathrm{mob}}=60 \mathrm{~nm}$ to $<400 \mathrm{~kg} \mathrm{~m}^{-3}$ at $D_{\mathrm{mob}}=700 \mathrm{~nm}$ (all data are also tabulated in the Supplement). Decreasing effective density with decreasing mobility diameter is consistent with aggregates of small irregular flakes of graphite (see Moteki et al., 2009, for a detailed characterisation of the structure of Aquadag). The black line is a polynomial fit through both PSI and UofA data, and the grey shading indicates $\pm 10 \%$ deviation from this fit, which corresponds to the estimated accuracy of the measurements. The measurements done by PSI and UofA agree within uncertainty across the whole common diameter range. Furthermore, no significant difference in effective density was observed between the Aquadag batches "classic" (diamonds) and "new bottle" (circles) by either investigator. The data series of UofA contains multiple data points at certain mobility diameters because additional effective density measurements were made from suspensions with doubled and halved Aquadag concentration (not distinguished in Fig. 3 for clarity reasons). Variation of suspension concentration has also no significant effect on the measured effective density. Recapitulating, the relationship between mobility diameter and effective density (and mass) of Aquadag particles seems to be largely independent 
Table 1. Polynomial coefficients for the mass, $m$ [fg], and effective density, $\rho_{\text {eff }}\left[\mathrm{kg} \mathrm{m}^{-3}\right]$, of Aquadag and fullerene soot as a function of mobility diameter, $D_{\text {mob }}[\mathrm{nm}]$. Warning: Do not round below coefficients to less significant digits.

\begin{tabular}{crrrrr}
\hline & \multicolumn{2}{c}{ Aquadag $^{\mathrm{a}}$} & & \multicolumn{2}{c}{ Fullerene soot ${ }^{\mathrm{b}}$} \\
\cline { 2 - 3 } \cline { 5 - 6 } & $m\left(D_{\mathrm{mob}}\right)^{\mathrm{c}}$ & $\rho_{\mathrm{eff}}\left(D_{\mathrm{mob}}\right)^{\mathrm{d}}$ & & $m\left(D_{\mathrm{mob}}\right)^{\mathrm{c}}$ & $\rho_{\mathrm{eff}}\left(D_{\mathrm{mob}}\right)^{\mathrm{d}}$ \\
\hline c0 & -0.096 & 1900 & & -0.298 & 2096 \\
c1 & 0.00422 & -25.959 & & 0.01055 & -24.952 \\
c2 & $-4.82 \mathrm{e}-5$ & 0.239644 & & $-1.071 \mathrm{e}-4$ & 0.191165 \\
c3 & $6.26 \mathrm{e}-7$ & $-1.21482 \mathrm{e}-3$ & & $8.97 \mathrm{e}-7$ & $-8.3812 \mathrm{e}-4$ \\
c4 & $-5.65 \mathrm{e}-10$ & $3.65087 \mathrm{e}-6$ & & $-1.441 \mathrm{e}-9$ & $2.27956 \mathrm{e}-6$ \\
c5 & $-1.99 \mathrm{e}-13$ & $-6.71352 \mathrm{e}-9$ & & $1.412 \mathrm{e}-12$ & $-4.00845 \mathrm{e}-9$ \\
c6 & $3.80 \mathrm{e}-16$ & $7.41508 \mathrm{e}-12$ & & $-8.53 \mathrm{e}-16$ & $4.57438 \mathrm{e}-12$ \\
c7 & - & $-4.51461 \mathrm{e}-15$ & & $2.16 \mathrm{e}-19$ & $-3.27578 \mathrm{e}-15$ \\
c8 & - & $1.16399 \mathrm{e}-18$ & & - & $1.33853 \mathrm{e}-18$ \\
c9 & - & - & & - & $-2.3817 \mathrm{e}-22$ \\
\hline
\end{tabular}

a Parametrizations for Aquadag are valid between $60 \mathrm{~nm}<D_{\text {mob }}<710 \mathrm{~nm}$. ${ }^{\text {b }}$ Parametrizations for fullerene soot are valid between $65 \mathrm{~nm}<D_{\text {mob }}<900 \mathrm{~nm}$. ${ }^{\mathrm{c}} m\left(D_{\mathrm{mob}}\right)=\sum_{i} c_{i} D_{\mathrm{mob}}^{i},{ }^{\mathrm{d}} \rho_{\mathrm{eff}}\left(D_{\mathrm{mob}}\right)=\sum_{i} c_{i} D_{\mathrm{mob}}^{i}$.

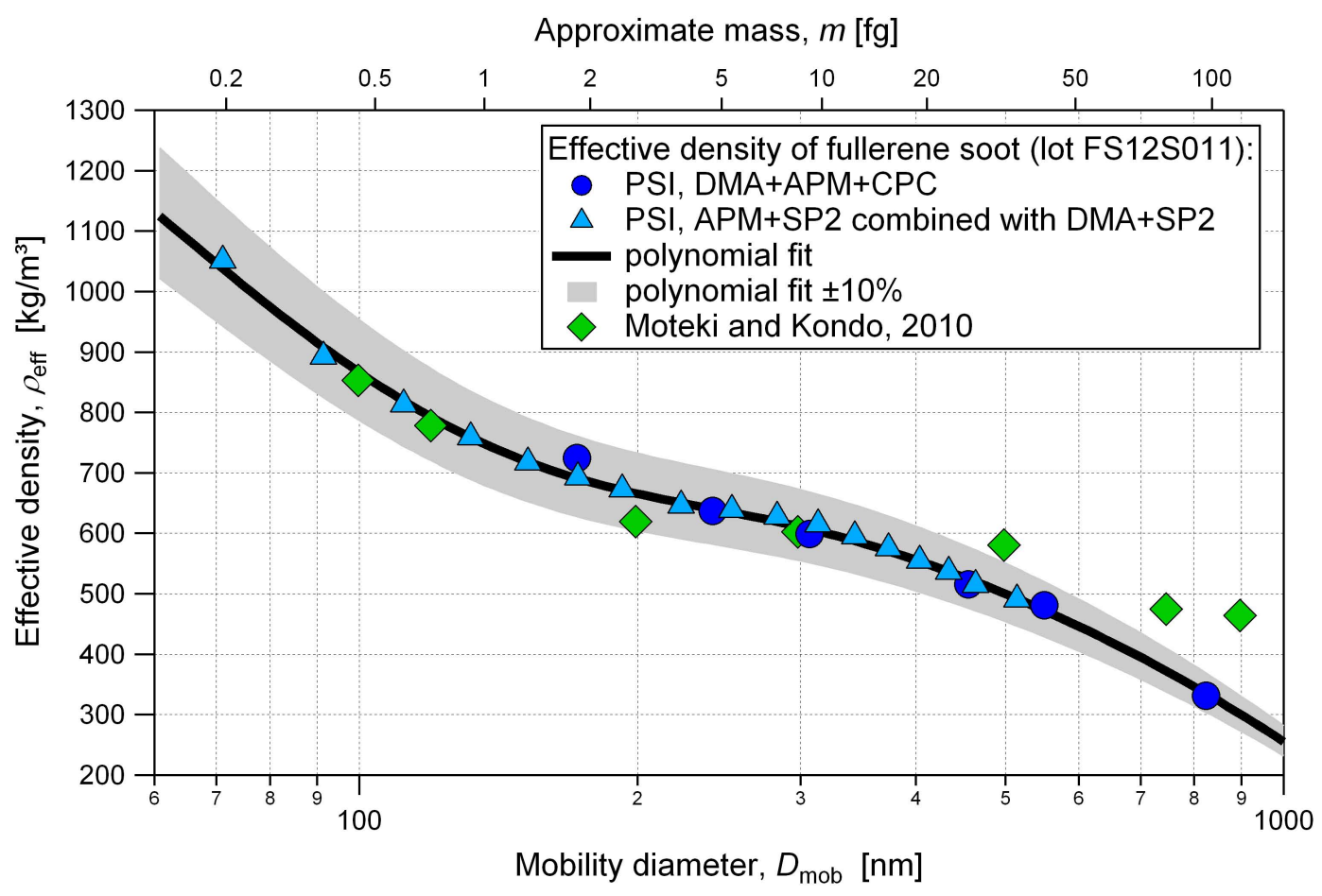

Fig. 4. Effective density of fullerene soot as a function of mobility diameter. The approximate mass scale given at the top axis has been calculated from the mobility diameters of the bottom axis using the polynomial fit.

of production lot and aerosol generation procedures. Thus it can be expected that the polynomial fit shown in Fig. 3 makes reproducible Aquadag based SP2 calibrations possible, even if only a DMA is available for selecting particles by their mobility diameter. The mass as a function of mobility diameter is either obtained by combining the parametrization of the effective density with Eq. (1) or from a direct parametrization of the mass. Polynomial coefficients of both parametrizations are provided in Table 1.

The effective density measurements of Aquadag, previously made by Moteki and Kondo (2010) using a similar DMA + APM setup, are added to Fig. 3 as green squares. Their results agree with this study within experimental uncertainty at mobility diameters $D_{\mathrm{mob}}<240 \mathrm{~nm}(m \approx 5 \mathrm{fg})$. 


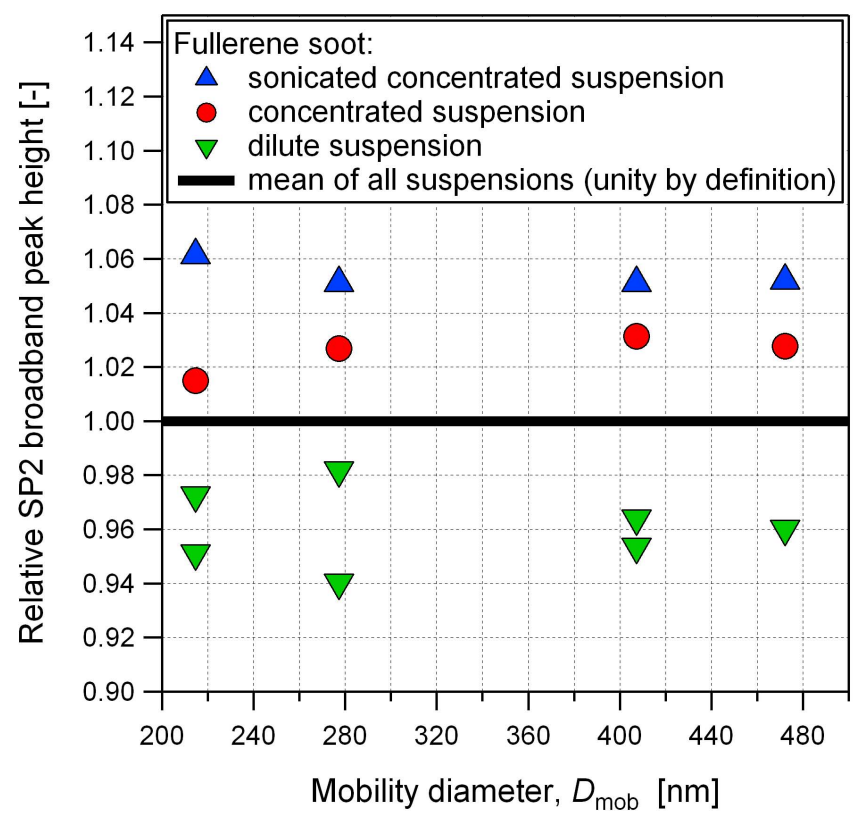

Fig. 5. Effect of different fullerene soot suspension treatments on the SP2 calibration based on particle selection by a DMA. The relative SP2 broadband peak height is obtained by division of the broadband peak height measured for a certain suspension by the mean value of the broadband peak heights measured for all suspensions at equal mobility diameter.

However, they reported higher effective density at larger mobility diameters with deviations of up to $\sim 35 \%$ in the range $D_{\text {mob }} \approx 500-750 \mathrm{~nm}$. The reason for this systematic difference is unclear, but a similiar deviation was also observed for fullerene soot as will be shown in Sect. 3.2. However, this difference at larger particle mass is of minor relevance for atmospheric measurements, as the BC mass size distribution typically peaks below $\sim 10$ fg BC per particle (e.g. Schwarz et al., 2010).

\subsection{Fullerene soot}

The effective density of one batch of fullerene soot was measured by PSI with two different methods (see Sect. 2.2). Figure 4 shows results from the "traditional" APM + DMA approach as dark blue circles and results from the novel indirect method as light blue triangles (all data are also tabulated in the Supplement). The black line is a polynomial fit through all data obtained from both methods, and the grey shading indicates $\pm 10 \%$ deviation from this fit, which corresponds to the estimated accuracy of the measurements.

Differences of effective density data measured by the two methods are well within experimental uncertainty, which shows that a DMA combined with an SP2 can be used for fast effective density measurements of pure BC particles. It has to be emphasized that this is only possible if an accurate calibration of the SP2 for the BC material in question has been done using an APM or CPMA, given the fact that the SP2's sensitivity differs substantially between different BC materials (Moteki and Kondo, 2010; Laborde et al., 2011b). The effective density of fullerene soot decreases from $\sim 1000 \mathrm{~kg} \mathrm{~m}^{-3}$ at $D_{\mathrm{mob}}=80 \mathrm{~nm}$ to $<400 \mathrm{~kg} \mathrm{~m}^{-3}$ at $D_{\text {mob }}>700 \mathrm{~nm}$. This is much lower than the bulk density of fullerene soot of $\sim 1720 \mathrm{~kg} \mathrm{~m}^{-3}$ (Kondo et al., 2011), and consistent with a fractal-like agglomerate of small primary spheres (see Moteki et al., 2009, for a detailed characterisation of the structure of fullerene soot).

The effective density measurements of fullerene soot, previously made by Moteki and Kondo (2010) using a similar DMA+APM setup, are added to Fig. 4 as green squares. Their results agree with this study within experimental uncertainty at mobility diameters $D_{\text {mob }}<440 \mathrm{~nm}(m \approx 4.5 \mathrm{fg})$. However, they reported higher effective density at larger mobility diameters with deviations of $20-30 \%$ in the range $D_{\text {mob }}=500-750 \mathrm{~nm}(m \approx 33-82 \mathrm{fg})$. This systematic difference is similar to the deviations observed for Aquadag at larger diameters. Careful analysis of the measurements done in this study gave no indication of any potential problem systematically occurring in the range of larger mobility diameters and higher mass. Hence the reason for the observed differences remains unclear. Anyway, this difference at larger particle mass is of minor relevance for atmospheric measurements, as the $\mathrm{BC}$ mass size distribution typically peaks below $\sim 10$ fg BC per particle (e.g. Schwarz et al., 2010).

The stability and reproducibility of the fullerene soot's effective density as well as of DMA based SP2 calibrations was investigated by measuring the mass of size-selected fullerene soot particles generated from different suspensions with the SP2. Three different aqueous suspensions of fullerene soot originating from the same production lot were produced: dilute suspension, concentrated suspension and sonicated concentrated suspension. The relative broadband incandescence peak height detected by the SP2 for all three suspensions is shown in Fig. 5 as a function of selected mobility diameter. "Relative peak height" means that the broadband peak height detected for a certain suspension and mobility diameter was divided by the mean peak height detected for all suspensions at the same mobility diameter. Differences between the three suspensions are as small as $\pm 6 \%$, which includes the variability of the effective density as well as the variability of the DMA and the SP2. Thus it can be expected that the polynomial fit shown in Fig. 4 makes reproducible fullerene soot based SP2 calibrations possible, even if only a DMA is available for selecting particles by their mobility diameter. Parametrizations of the effective density and mass of fullerene soot as a function of mobility diameter are provided in Table 1. 


\section{Conclusions}

The mass and effective density of Aquadag and fullerene soot particles generated from aqueous suspensions were measured as a function of their mobility diameter by operating a DMA and an APM or CPMA in series. Consistent and reproducible results were found for Aquadag by two research groups for different production lots and suspension treatments. The effective density of fullerene soot particles was also found to be independent of suspension treatments. Only one production lot has been tested and thus generalisation to any other production lot of fullerene soot is not possible. This study shows that the effective density of Aquadag and fullerene soot particles is a stable quantity and largely unaffected by differences in aerosol generation procedures. Stability and knowledge of the effective density of BC reference materials makes reproducible SP2 calibrations possible if only a DMA is available for size-selection of the BC particles.

Additional tests revealed that the Aquadag and fullerene soot calibration particles generated by atomization and diffusion drying contain substantial amounts of non-EC components. This raises the question whether the SP2 calibration curves should be corrected for the mass of the non-EC components. The SP2's sensitivity to pure ambient $\mathrm{BC}$ compared to untreated and thermodenuded Aquadag and fullerene soot was investigated in a follow-up study by Laborde et al. (2011b). They concluded that the calibration standards should be used in the untreated form. Furthermore, fullerene soot has been suggested as a preferred calibration standard for ambient purposes, in agreement with previous results for ambient $\mathrm{BC}$ in Tokyo reported by Moteki and Kondo (2010). The accuracy of SP2 measurements depends both on using a calibration standard suitable for the investigated samples and on using correct effective density data for the evaluation of DMA-based SP2 calibrations.

\section{Supplementary material related to this article is available online at: http://www.atmos-meas-tech.net/4/2851/2011/ amt-4-2851-2011-supplement.zip.}

Acknowledgements. We thank J. P. Schwarz from NOAA for providing a batch of fullerene soot for instrument calibration and effective density measurements. S. E. Pratsinis is acknowledged for granting access to his APM. Financial support was received from the Swiss National Science Foundation. J. S. Olfert would like to thank Cambustion Ltd. for providing support for the construction of the CPMA used in this study.

Edited by: A. Wiedensohler

\section{References}

Cross, E. S., Onasch, T. B., Ahern, A., Wrobel, W., Slowik, J. G., Olfert, J., Lack, D. A., Massoli, P., Cappa, C. D., Schwarz, J. P., Spackman, J. R., Fahey, D. W., Sedlacek, A., Trimborn, A., Jayne, J. T., Freedman, A., Williams, L. R., Ng, N. L., Mazzoleni, C., Dubey, M., Brem, B., Kok, G., Subramanian, R., Freitag, S., Clarke, A., Thornhill, D., Marr, L. C., Kolb, C. E., Worsnop, D. R., and Davidovits, P.: Soot particle studies - Instrument inter-comparison - project overview, Aerosol Sci. Technol., 44, 592-611, doi:10.1080/02786826.2010.482113, 2010.

DeCarlo, P. F., Slowik, J. G., Worsnop, D. R., Davidovits, P., and Jimenez, J. L.: Particle morphology and density characterization by combined mobility and aerodynamic diameter measurements. Part 1: Theory, Aerosol Sci. Technol., 38, 1185-1205, 2004.

Ehara, K., Hagwood, C., and Coakley, K. J.: Novel method to classify aerosol particles according to their mass-to-charge ratio Aerosol particle mass analyser, J. Aerosol Sci., 27, 217-234, doi:10.1016/0021-8502(95)00562-5, 1996.

Gelencsér, A.: Carbonaceous Aerosol, vol. 30 of Atmospheric and Oceanographic Sciences Library, Springer Netherlands, Dordrecht, doi:10.1007/978-1-4020-2887-8, 2004.

Knutson, E. and Whitby, K. T.: Aerosol classification by electric mobility: Apparatus, theory, and applications, J. Aerosol Sci., 6, 443-451, doi:10.1016/0021-8502(75)90060-9, 1975.

Kondo, Y., Sahu, L., Moteki, N., Khan, F., Takegawa, N., Liu, X., Koike, M., and Miyakawa, T.: Consistency and traceability of black carbon measurements made by laser-induced incandescence, thermal-optical transmittance, and filter-based photo-absorption techniques, Aerosol Sci. Technol., 45, 295312, doi:10.1080/02786826.2010.533215, 2011.

Laborde, M., Gysel, M., and the SOOT11 TEAM: Calibration and intercomparison study of 6 SP2 investigating different types of soot particles and black carbon reference materials, manuscript in preparation, 2011a.

Laborde, M., Mertes, P., Zieger, P., Dommen, J., Baltensperger, U., and Gysel, M.: Sensitivity of the Single Particle Soot Photometer to different black carbon types, Atmos. Meas. Tech. Discuss., submitted, $2011 \mathrm{~b}$.

Laden, F., Neas, L. M., Dockery, D. W., and Schwartz, J.: Association of fine particulate matter from different sources with daily mortality in six US cities, Environ. Health Perspect., 108, 941947, 2000.

Moteki, N. and Kondo, Y.: Effects of mixing state on black carbon measurements by laser-induced incandescence, Aerosol Sci. Technol., 41, 398-417, doi:10.1080/02786820701199728, 2007.

Moteki, N. and Kondo, Y.: Dependence of laser-induced incandescence on physical properties of black carbon aerosols: measurements and theoretical interpretation, Aerosol Sci. Technol., 44, 663-675, doi:10.1080/02786826.2010.484450, 2010.

Moteki, N., Kondo, Y., Takegawa, N., and Nakamura, S.: Directional dependence of thermal emission from nonspherical carbon particles, J. Aerosol Sci., 40, 790-801, doi:10.1016/j.jaerosci.2009.05.003, 2009.

Moteki, N., Kondo, Y., and Nakamura, S.: Method to measure refractive indices of small nonspherical particles: Application to black carbon particles, J. Aerosol Sci., 41, 513-521, doi:10.1016/j.jaerosci.2010.02.013, 2010. 
Olfert, J. S. and Collings, N.: New method for particle mass classification - the Couette centrifugal particle mass analyzer, J. Aerosol Sci., 36, 1338-1352, doi:10.1016/j.jaerosci.2005.03.006, 2005.

Olfert, J. S., Symonds, J. P. R., and Collings, N.: The effective density and fractal dimension of particles emitted from a lightduty diesel vehicle with a diesel oxidation catalyst, J. Aerosol Sci., 38, 69-82, doi:10.1016/j.jaerosci.2006.10.002, 2007.

Ramanathan, V. and Carmichael, G.: Global and regional climate changes due to black carbon, Nat. Geosci., 1, 221-227, doi:10.1038/ngeo156, 2008.

Scheckman, J. H., McMurry, P. H., and Pratsinis, S. E.: Rapid characterization of agglomerate aerosols by in situ mass-mobility measurements, Langmuir, 25, 8248-8254, doi:10.1021/la900441e, 2009.

Schwarz, J. P., Gao, R. S., Fahey, D. W., Thomson, D. S., Watts, L. A., Wilson, J. C., Reeves, J. M., Darbeheshti, M., Baumgardner, D. G., Kok, G. L., Chung, S. H., Schulz, M., Hendricks, J., Lauer, A., Kärcher, B., Slowik, J. G., Rosenlof, K. H., Thompson, T. L., Langford, A. O., Loewenstein, M., and Aikin, K. C.: Single-particle measurements of midlatitude black carbon and light-scattering aerosols from the boundary layer to the lower stratosphere, J. Geophys. Res., 111, D16207, doi:10.1029/2006JD007076, 2006.
Schwarz, J. P., Spackman, J. R., Gao, R. S., Watts, L. A., Stier, P., Schulz, M., Davis, S. M., Wofsy, S. C., and Fahey, D. W.: Global-scale black carbon profiles observed in the remote atmosphere and compared to models, Geophys. Res. Lett., 37, L18812, doi:10.1029/2010GL044372, 2010.

Slama, R., Morgenstern, V., Cyrys, J., Zutavern, A., Herbarth, O., Wichmann, H. E., Heinrich, J., and Group, L. S.: Trafficrelated atmospheric pollutants levels during pregnancy and offspring's term birth weight: a study relying on a land-use regression exposure model, Environ. Health Perspect., 115, 12831292, doi:10.1289/ehp.10047, 2007.

Slowik, J. G., Cross, E. S., Han, J.-H., Davidovits, P., Onasch, T. B., Jayne, J. T., Williams, L. R., Canagaratna, M. R., Worsnop, D. R., Chakrabarty, R. K., Moosmüller, H., Arnott, W. P., Schwarz, J. P., Gao, R.-S., Fahey, D. W., Kok, G. L., and Petzold, A.: An inter-comparison of instruments measuring black carbon content of soot particles, Aerosol Sci. Technol., 41, 295314, doi:10.1080/02786820701197078, 2007.

Stephens, M., Turner, N., and Sandberg, J.: Particle identification by laser-induced incandescence in a solid-state laser cavity, Appl. Opt., 42, 3726-3736, doi:10.1364/AO.42.003726, 2003. 